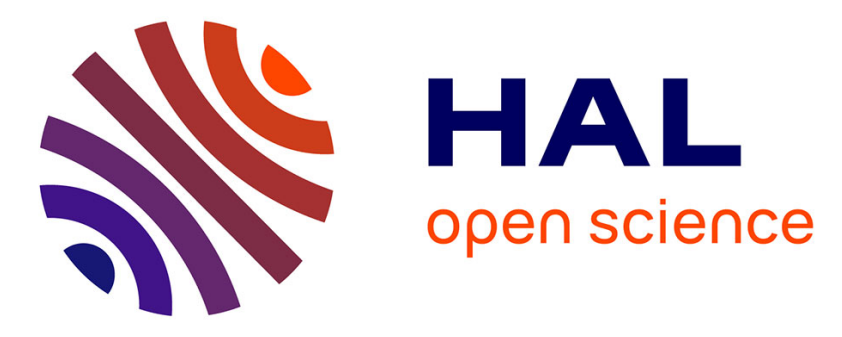

\title{
La formation d'une culture régionale en Djézireh, le témoignage d'une tombe de guerrier de la période amorrite
}

Christophe Nicolle

\section{- To cite this version:}

Christophe Nicolle. La formation d'une culture régionale en Djézireh, le témoignage d'une tombe de guerrier de la période amorrite. Grégory Chambon., Michaël Guichard, Anne-Isabelle Langlois (éditeurs). De l'argile au numérique. Mélanges assyriologiques en l'honneur de Dominique Charpin, Peeters, pp.739-762, 2019, Publications de l'Institut du Proche-Orient Ancien du Collège de France, 3, 978-90-429-3872-4. hal-02440644

\section{HAL Id: hal-02440644 \\ https://hal.science/hal-02440644}

Submitted on 15 Jan 2020

HAL is a multi-disciplinary open access archive for the deposit and dissemination of scientific research documents, whether they are published or not. The documents may come from teaching and research institutions in France or abroad, or from public or private research centers.
L'archive ouverte pluridisciplinaire HAL, est destinée au dépôt et à la diffusion de documents scientifiques de niveau recherche, publiés ou non, émanant des établissements d'enseignement et de recherche français ou étrangers, des laboratoires publics ou privés. 
This pdf is a digital offprint of your contribution in G. Chambon, M. Guichard \& A.-I. Langlois (eds), De l'argile au numérique. Mélanges assyriologiques en l'honneur de Dominique Charpin, ISBN 978-90-429-3872-4.

The copyright on this publication belongs to Peeters Publishers.

As author you are licensed to make printed copies of the pdf or to send the unaltered pdf file to up to 50 relations. You may not publish this pdf on the World Wide Web including websites such as academia.edu and open-access repositories - until three years after publication. Please ensure that anyone receiving an offprint from you observes these rules as well.

If you wish to publish your article immediately on openaccess sites, please contact the publisher with regard to the payment of the article processing fee.

For queries about offprints, copyright and republication of your article, please contact the publisher via peeters@peeters-leuven.be 
PIPOAC 3

\title{
DE L'ARGILE AU NUMÉRIQUE
}

\author{
MÉLANGES ASSYRIOLOGIQUES \\ EN L'HONNEUR \\ DE DOMINIQUE CHARPIN
}

édités par

Grégory Chambon, Michaël Guichard et Anne-Isabelle Langlois

avec la participation de

Thomas Römer et Nele Ziegler

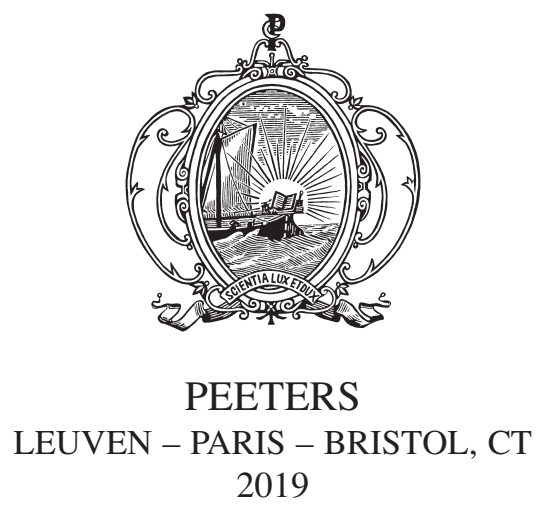




\section{TABLE DES MATIÈRES}

Avant-propos................... $\mathrm{V}$

Bibliographie de Dominique Charpin.......... VII

Abdallah, F., Dynasties du Yamhad $($ Halab = Alep $) .. . . . \quad 1$

Alexandrov, B., Posing a Question in Akkadian: Wh- and Other Interrogatives in Old Babylonian Letters. . . . . . . . 13

ARKHIPOV, I., Ittum « signe » et ittum « moment » en paléobabylonien ..................... 47

Attinger, P., Našše A. . . . . . . . . . . . . . . . 79

BÉRANGER, M., Du signe à l'enveloppe. L'enseignement du genre épistolaire à l'époque amorrite d'après un nouveau modèle de lettre scolaire avec enveloppe . . . . . . . . . . 125

BeYER, D., Une empreinte de sceau-cylindre du palais de Mari . 169

CANCIK-KIRSChBAUM, E., ${ }^{\mathrm{D}} \mathrm{GU}_{4}$-DUMU-DUTU in Assur in mittelassyrischer Zeit . . . . . . . . . . . . . 175

Caubet, A. \& Yon, M., Importation asiatique en Grèce mycénienne. La « Combat Agate» de Pylos . . . . . . . . . . . 189

Chalendar, V., Un serment dur à avaler, les manifestations pathologiques du serment. . . . . . . . . . 203

Chambon, G. \& Guichard, M., Le monde en tableaux (I) : une étude des tablettes-registres de la grande fête d'Eštar à Mari . . . . . . . . . . . . . . . . . . . 225

CoHEN, Y., Forging an Empire: The Land of Aštata According to the Šattiwaza Treaty (CTH 51) and Additional Sources. . .

Cousin, L., Entre Ur et Eridu : réflexions autour d'une brique d'Amar-Sin . . . . . . . . . . . . . . . 299

DE BOER, R., Pīhatni-ipiq, an official in the service of king Sumu-El of Larsa . . . . . . . . . . . . . . . . . . 307

Delnero, P., Sound and Meaning in Sumerian Cultic Laments . 315

DÉmARE-LAFOnt, S., Équité et légalité à Emar . . . . . . . . . . 357

DurAnd, J.-M., Un joint dans les textes de Mari. . . . . . . . . 373 
Fiette, B., Des bateaux pour Babylone. . . . . . . . . . . . . .

Fleming, D., Only the maš'artu-Priestess Speaks: The Emar Installation Rites in Scribal Context. . . . . . . . . . . . .

Foster, B., Language Study and the Beginnings of the Collège de France . . . . . . . . . . . . . . . . . 425

HeEssel, N., Träum was Schönes! . . . . . . . . . . . . . . . . 441

Huot, J.-L., Le mystère des remparts de Larsa. . . . . . . . . . 455

JACQUET, A. \& RECULEAU, H., Tablettes paléo-babyloniennes de la collection R. F. Harper conservées à l'Oriental Institute de l’Université de Chicago . . . . . . . . . . . . . . . . . . 469

JoAnNÈs, F., « Mettez-vous en quête de ce sceau ! » . . . . . . 491

Jursa, M., A 'Prisoner Text' from Birmingham . . . . . . . . . 507

Lacambre, D., Samsī-Addu et la constitution de son empire : nouvelles données. . . . . . . . . . . . . . . . . . 513

LANGLOIS, A.-I., Quelques ânes en plus... . . . . . . . . . . . 545

Lauinger, J., Contribution to a Diplomatics of the Amarna Letters from the Levant: « la mise en page » . . . . . . . . . 563

Lion, B., Mariages et archives dans le Royaume d'Arraphe . . . 583

LyONNET, B., La « céramique du Khabur » et ses relations orientales : les routes de l'étain . . . . . . . . . . . . . . . . 613

Marti, L., Les archives de Tell Munbâqa : le cas de la maison B . . . . . . . . . . . . . . . . . . . . 639

MAUL, S., Eidesleistung und Selbstverfluchung. Überlegungen zu KAR 373 (VAT 8719) . . . . . . . . . . . . . . . . 655

Michalowski, P., Memories of Rim-Sin II . . . . . . . . . . . 669

MolinA, M., Sumerian Judicial Procedures against Tomb Robbers 693

NeBIOLO, F., Iprum, iptirum ed altre richieste: una lettera inedita dalla collezione dell'Iraq Museum di Baghdad . . . . . . . .

Neumann, H., Bemerkungen zur Identität und Rolle des qajjāp(ān)um in $\S 5 / 6$ des Edikts Ammi-șaduqa. . . . . . .

NiColle, C., La formation d'une culture régionale en Djézireh, le témoignage d'une tombe de guerrier de la période amorrite

Отто, A., Official Seal Motifs at Larsa and Ur in the $19^{\text {th }}$ Century BC. . . . . . . . . . . . . . . . . . .

Owen, D., 'Death for Default' Redux. Akkadian Field Cultivation and Other Akkadian Contracts from Iri-Saĝrig . . . . . . 
Parpola, S., The Sumerian $2^{\text {nd }}$ Person Pronoun, and Latin and French Morphophonemics . . . . . . . . . . . . . .

Pozzer, K., Les divinités au féminin - une étude des sceauxcylindres mésopotamiens . . . . . . . . . . . . .

RADNER, K., Mistakes were Made ... on the Throne base of Shalmaneser III of Assyria . . . . . . . . . . . . . . .

RAmez, M., «Tu représenteras parfaitement la force de mon héroïsme ! » La diorite comme vecteur de l'idéologie royale au pays de Sumer et d'Akkad (III ${ }^{\mathrm{e}}-\mathrm{II}{ }^{\mathrm{e}}$ millénaires av. J.-C.)

Rотн, M., Seductress or Victim? Another Look at Laws of Ur-Namma $\S \S 6-8$.

Sallaberger, W., Who Is Elite? Two Exemplary Cases from Early Bronze Age Syro-Mesopotamia . . . . . . . . . . . 893

SASSON, J., Vile Threat: The Rhetoric of a Marital Spat . . . . . 923

Shibata, D., The Gods of Tabetu during the Middle Assyrian Period and their Genealogy. . . . . . . . . . . .

SteinKeller, P., Texts, Art and Archaeology: An Archaic Plaque from Mari and the Sumerian Birth-Goddess Ninhursag . . .

Stol, M., Old Babylonian kišdātum, "what one may get”. . . .

TANRET, M., Gimil-Marduk. Une carrière de juge ou un juge de carrière ? . . . . . . . . . . . . . . . . . 1031

Thomas, A., L'adorant de Larsa au Louvre. . . . . . . . . . . 1059

van Koppen, F., Itur-Asdu of River Silakku. A Contribution to the Kingship of Hammurabi of Babylon . . . . . . . . . . . 1081

VAn Lerberghe, K., Abiešuh's Year Four . . . . . . . . . . . . 1103

Veldhuis, N., Word Studies: Ur III and ePSD2 . . . . . . . . . 1113

WASSERMAN, N., Mesopotamian Underwear and Undergarments 1125

Weszeli, M., Two Dossiers of Boat Owners and Hirers of Small Watercraft from the Area of Yahrurum šaplûm . . . . . . . 1145

WiLheLM, G., Mari hurr. Nr. 8 . . . . . . . . . . . . . . . 1181

YamadA, S., Sim'alites at Țabatum and the Origin of the Kingdom of the "Land of Hana" . . . . . . . . . . . . . . . 1189

ZGOLL, A., Mythos als rituell aufgeführtes Drama. Inthronisation, Tempelschöpfung und Stadtgründung im altbabylonischen Lied auf Bazi . . . . . . . . . . . . . . . . . . . . . . . 1209

ZIEGLER, N., La qadištum dépouillée . . . . . . . . . . . . . . 1243 


\title{
LA FORMATION \\ D'UNE CULTURE RÉGIONALE EN DJÉZIREH, LE TÉMOIGNAGE D'UNE TOMBE DE GUERRIER DE LA PÉRIODE AMORRITE
}

\author{
Christophe NICOLLE*
}

Entre 2150-2000 av. J.-C. la région du triangle du Khabour traverse une période de récession de son occupation marquée par l'abandon ou un repli important de nombreuses villes accompagnant une disruption de sa culture régionale. À cet épisode encore mal documenté, succède une phase de réoccupation, elle aussi encore obscure. Elle correspond à un renouvellement important de la population et la mise en place d'un nouveau réseau d'agglomérations. Elle apparaît liée à d'importants mouvements de populations perceptibles dans la Mésopotamie et le Levant. En Mésopotamie, le phénomène est repérable par l'installation de nombreux dynastes portant des noms amorrites. L'arrivée de ce groupe ethnique est considérée tout à la fois comme la cause de l'effondrement des cadres politiques antérieures, de leur renouvellement et de leur revitalisation. L'avancée des recherches et de nouvelles découvertes archéologiques montrent qu'il convient de repenser la place tenue par l'ethnie amorrite dans la phase de recomposition du paysage politique du début du $\mathrm{II}^{\mathrm{e}}$ millénaire. De fait, l'influence et le rôle de cette ethnie doivent être considérés en tenant compte de l'existence d'autres ethnies elles aussi présentent dans la région. Il en est notamment de l'ethnie hourrite dont plusieurs dirigeants sont à la tête de différents royaumes durant le dernier tiers du III ${ }^{\mathrm{e}}$ millénaire. Les ferments et les origines de la nouvelle culture régionale qui se met en place et se développe dans le triangle du Khabour durant la première moitié du $\mathrm{II}^{\mathrm{e}}$ millénaire apparaissent ainsi multiples. Ils sont d'autant plus difficiles à saisir. Lors de la dernière campagne de fouille menée en 2010, à Tell Mohammed Diyab, une importante agglomération du Bronze moyen, une intéressante découverte a été faite. Il s'agit d'une tombe dite « de guerrier». Par les connexions culturelles dont elle témoigne avec le monde levantin comme avec la zone du Nord mésopotamien, elle documente de manière utile la multiplicité des 
influences culturelles en action dans la composition d'une nouvelle culture régionale en Djézireh pendant la première moitié du $\mathrm{II}^{\mathrm{e}}$ millénaire. Le contexte de sa découverte et son contenu permettent d'envisager l'hypothèse qu'il s'agissait de la tombe d'un haut personnage, peut-être celle d'un marchand assyrien. Elle vient en tout cas enrichir le débat sur l'existence et les composantes d'une « koinè amorrite».

\section{LA DESCRIPTION DE LA TOMBE 5851}

La tombe 5851 a été découverte sur le sommet de la butte B où se trouvent concentrés plusieurs bâtiments importants dont un petit palais et plusieurs entrepôts de la période céramique Khabour reconstruits à plusieurs reprises durant les niveaux 6b-8, 7 et 6. À l'emplacement du chantier $6 b^{1}$, un des quatre chantiers ouverts sur la butte $B$, les découvertes signalent une occupation marquée par plusieurs ruptures et de nombreuses reconstructions. C'est durant l'une de ces périodes d'abandon à une date encore imprécise, sans doute au début de la période OJZ $2^{2}$ que se place la construction de la tombe du guerrier (niveau 6b-7) dans les ruines d'un premier niveau d'entrepôts (niveau 6b-8). Bien qu'encore mal datée, sa position stratigraphique est sans équivoque. Le dégagement de sa fosse montre que son creusement a coupé un mur arasé du niveau 6b-8 (M.5656) au moins à partir de la cote de 396,55 m. L'ouverture de la fosse fut par la suite scellée par la construction d'un autre mur (M.4292) d'un entrepôt construit au niveau 6b-6 (période OJZ 2).

L'épisode de la tombe se compose d'un ensemble de faits : le creusement d'une fosse (fosse 5761), la construction de la tombe 5851, l'inhumation du défunt, le bouchage de la tombe, le sacrifice d'un équidé et le comblement de la fosse (Fig. 1).

De forme rectangulaire $(2,70 \mathrm{~m} \times 1,70 \mathrm{~m})^{3}$ avec une orientation nordest/sud-ouest, la fosse aux parois verticales était remplie d'un limon gris cendreux à faible granulométrie. Le fond de la fosse a été atteint $1,90 \mathrm{~m}$ plus bas à la cote de $394,50 \mathrm{~m}$. Elle contenait une tombe rectangulaire en

1 Nicolle 2012.

2 Datation régionale usitée pour la région de la Djézireh ; Old Jezirah 2 (= OJZ 2) ca. 1850-1700 av. J.-C.

${ }^{3}$ Pour des raisons de sécurité et du fait de la présence au sud d'un mur plus tardif (M.4885) dont la base est constituée de gros blocs de basalte, la fosse n'a pas été complètement dégagée. Sa longueur totale est estimée à $3,10 \mathrm{~m}$. 
briques crues (locus 5851) avec une chambre funéraire de 1,20 m estouest et de 1,85 m nord-sud ${ }^{4}$. Ses parois sont formées par des murs composés d'une rangée de briques crues grises ${ }^{5}$ plus ou moins bien conservées et disposées sur 6, 7 assises. Des briques orangées de dimensions similaires constituent la partie médiane du mur nord. Elles signalent l'emplacement de l'accès à la tombe, bouché une fois l'inhumation réalisée. La couverture de la chambre funéraire partiellement conservée (avec un sommet à la cote de 395,20 m) à l'extrémité sud était composée de briques crues disposées de chant en "diamant ${ }^{6}$. Quelques pierres éparses posées à plat constituent le sol de la chambre funéraire. Dans la partie nord de la fosse, les constructeurs de la tombe avaient laissé un espace libre faisant office de puits d'accès ${ }^{7}$.

L'espace sépulcral contenait les restes d'un individu (5851-S-1) en décubitus latéral droit, jambes repliées et bras fléchis, la tête au sud regardant vers l'est. La présence d'une fine couche de poudre blanche sur ses os signale la présence d'un linceul composé d'une matière organique indéterminée.

Dans l'espace sépulcral tout autour du corps plusieurs objets en bronze étaient disposés : une hache fenestrée, deux lances, un poignard, une spatule, deux bols. Il y avait en outre une coupe carénée, un petit bol, une jarre les trois en terre cuite et des restes d'animaux (Fig. 2). Une seconde jarre de type Khabour était calée dans l'espace du puits d'accès sur le côté est de l'entrée de la tombe. La hache fenestrée était posée derrière le dos du mort à côté de deux javelines. Seule sa tête en bronze subsiste ainsi que des restes du manche en bois ${ }^{8}$. La hache appartient à un type d'arme bien documenté dont la zone principale de diffusion est la façade levantine, des plaines côtières aux vallées de l'Oronte et du Jourdain. Sa période d'utilisation est le Bronze moyen I levantin (2050-1800/1750 av. J.-C.). Si le groupe numériquement le plus important a été retrouvé sur

${ }^{4}$ Dimensions internes.

5 Dimensions des briques : $40 \times 40 \times 10 \mathrm{~cm}$.

${ }^{6} \mathrm{Ou}$ « briques de chant posées sur pointe » selon une typologie des tombes proposées par Ö. Tunca 2012, p. 140, fig. 15. Il s'agit d'un type de tombe que l'on retrouve au Bronze moyen sur d'autres sites de la région comme Tell Arbid ou Tell Chagar Bazar avec des variations dans les profondeurs d'enfouissement et la forme des fosses.

7 D'une largeur de $45-55 \mathrm{~cm}$.

${ }^{8}$ La lame de la hache (5851-M-5) est dotée de deux fenestrelles symétriques disposées de part et d'autre de l'axe médian de la lame. Le manche en bois était inséré dans le talon de la lame pourvue d'une douille de section ovale. Il ne subsiste que dans la douille. D'un poids de 270 gr, cette lame a une longueur de $10,7 \mathrm{~cm}$ pour une hauteur de $7,3 \mathrm{~cm}$. 

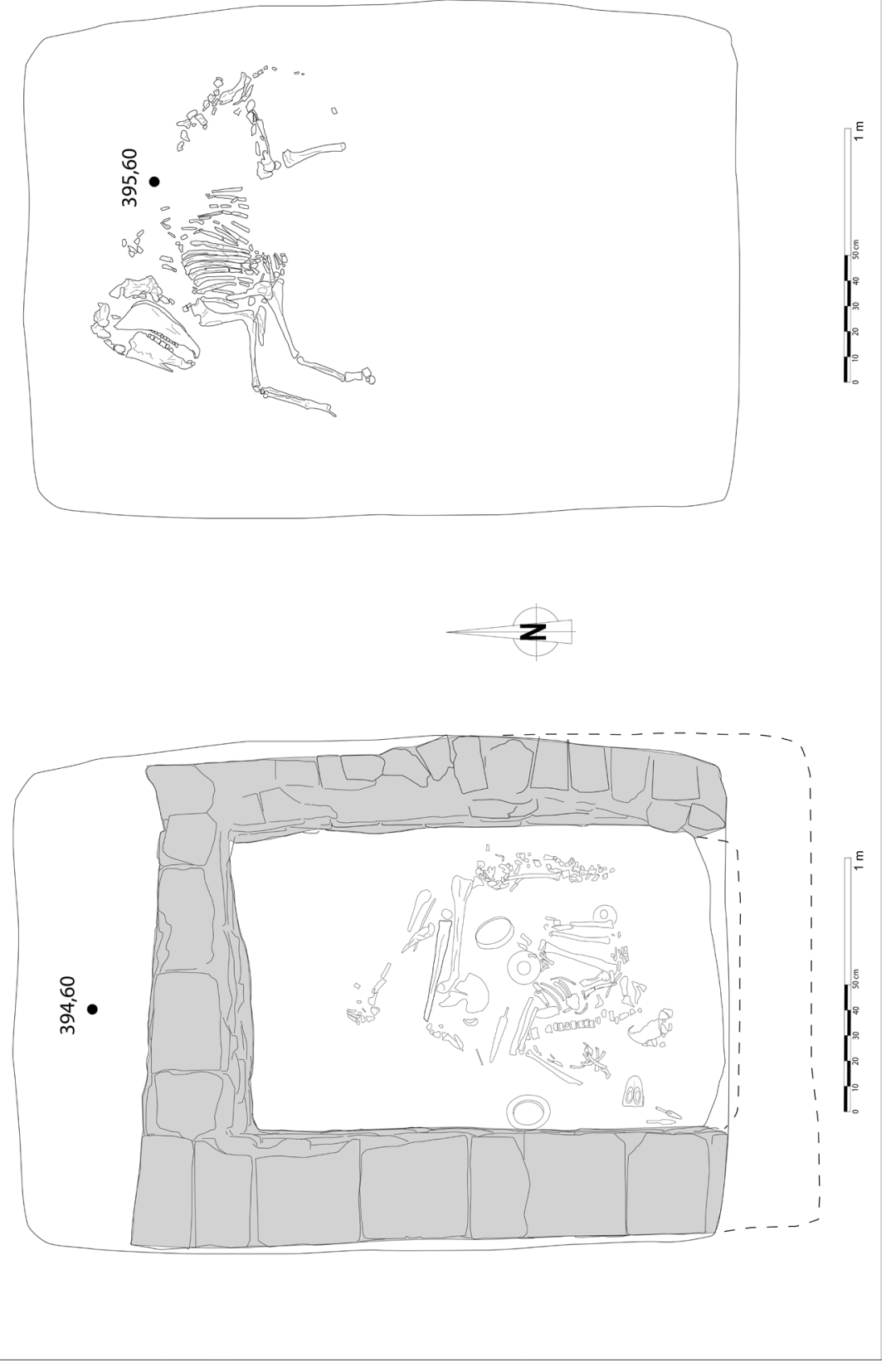

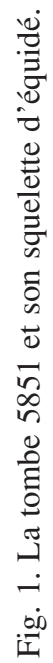




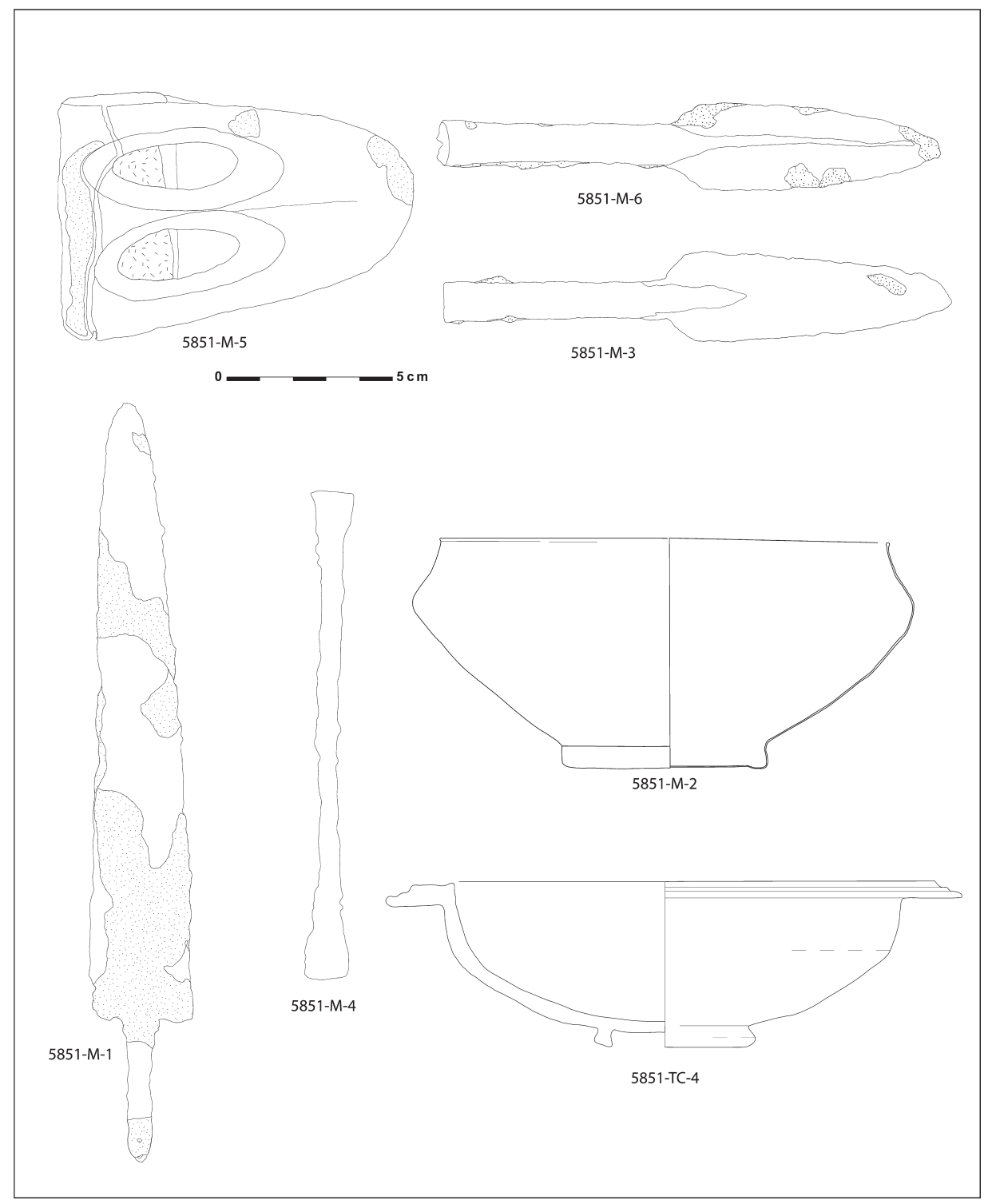

Fig. 2. Le matériel de la tombe 5851. 
la côte à Byblos 9 , neuf d'entre elles ont été retrouvées dans la nécropole de Baghouz sur les rives du moyen Euphrate syrien ${ }^{10}$.

Disposées à proximité de la hache, contre le mur ouest de la tombe, les deux pointes de lance sont de forme similaire. Il s'agit de lances à douille repliée de section circulaire et lame triangulaire, une forme d'emmanchement très répandue au Proche-Orient. Les pointes de lance sont de petites dimensions ${ }^{11}$, ce qui en fait des lances courtes, des armes de jet, sans doute des javelines. De légères différences notamment dans la longueur des douilles ${ }^{12}$ et dans la forme des pointes indiquent qu'elles n'ont pas été coulées dans le même moule ${ }^{13}$. Ce type de pointes de lance est largement répandu à partir de 2000 av. J.-C. à l'ensemble du Levant. Elles restent toutefois relativement rares en Mésopotamie. Un exemplaire similaire est connu à Tell Yelkhi dans la vallée du Hamrin, de plus nombreux dans la moyenne vallée de l'Euphrate syrien principalement à Baghouz ${ }^{14}$.

La quatrième arme découverte dans la tombe est un poignard dont seule subsistait la lame plate triangulaire prolongée de la partie d'emmanchement en bronze retrouvée au niveau de la hanche du squelette ${ }^{15}$. La partie de l'emmanchement est constituée d'une soie. La grande diffusion de ce type de poignard à soie rend difficile une délimitation spatio-temporelle précise. Un groupe mésopotamien apparaît à la fin de l'époque Isin Larsa ou au début de l'époque paléo-babylonienne ${ }^{16}$. Des exemples sont connus dans des tombes d'Assur ${ }^{17}$ et à Tell Abu Husseini ${ }^{18}$.

La tombe de Tell Mohammed Diyab contenait d'autres objets en bronze : une spatule en bronze ${ }^{19}$ retrouvée à proximité du poignard et

${ }^{9}$ La découverte de plusieurs exemplaires en or ou en argent découverts à Byblos dans des dépôts d'offrande et de fondation qui signalent que ce type d'arme pouvait acquérir un statut d'objet de prestige.

10 Gernez 2007, p. 195.

11 5851-M-3 et 6 . Elles mesurent $15,2 \mathrm{~cm}$ pour un poids de $50 \mathrm{gr}$ chacune et une épaisseur de la pointe de $0,7 \mathrm{~cm}$.

12 Une longueur de douille de 7,4 cm pour 5851-M-6 et de 6,6 $\mathrm{cm}$ pour 5851-M-3.

${ }^{13}$ Les restes des manches en bois subsistaient dans les douilles avec les rivets de fixation encore en place.

14 Vingt trois exemplaires ont été retrouvés dans la nécropole de Baghouz, cf. Mesnil du Buisson 1948 dans l'inventaire des tombes de la section Z, p. 63-93.

15 5851-M-1. L'ensemble d'une longueur $23,8 \mathrm{~cm}$ pour une largeur maximale de $23,8 \mathrm{~cm}$ pèse $85 \mathrm{gr}$.

${ }^{16}$ Gernez 2007, p. 450, son type P1b.

17 Haller 1954, tableau 7.

18 Müller-Karpe 2004, p. 1, pl. 12.

19 5851-M-4. D'une longueur de 14,6 cm et d'un poids de $15 \mathrm{gr}$. 
surtout deux exemplaires d'un même type de bols carénés en bronze ${ }^{20}$ déposés entre le torse du mort et ses cuisses. Les deux bols ressemblent fortement à une série des petits bols carénés peints en céramique de type Khabour que l'on voit apparaître au début du $\mathrm{II}^{\mathrm{e}}$ millénaire et qui disparaissent ensuite assez rapidement ${ }^{21}$.

Outre ces différents armes et objets en bronze qui représentent un total de 750 grammes de bronze, trois céramiques venaient compléter le dépôt funéraire. La première est une coupe à fond annulaire et pâte fine beige dotée d'une curieuse lèvre plate débordante ornée de deux cercles de peinture marron séparés par deux cercles d'incision ${ }^{22}$. Si le décor peint orangé est caractéristique de la première phase de la période Khabour, la forme de la coupe n'a pour le moment pas de parallèle connu. Au niveau de la main droite du mort il y avait une coupelle en terre cuite noire (5851-TC-3) sans doute façonnée à la main. Elle est elle aussi sans parallèle connu. Dans l'angle nord-est de la tombe, largement détruite par l'effondrement de la voûte de la tombe, il y avait les restes d'une grande jarre globulaire non peinte. Le long de la paroi est de la tombe avait été déposé un morceau de carcasse d'animal en connexion composé de la colonne vertébrale et un membre inférieur ${ }^{23}$. Une fois la dépose du mort, du matériel funéraire et des offrandes, l'accès à la tombe fut bloqué par des briques jaunes et le puits d'accès comblé. Le remplissage contenait plusieurs tessons khabour peint ${ }^{24}$.

Le comblement de la fosse fut interrompu $40 \mathrm{~cm}$ au-dessus de la couverture de la tombe à la cote 395,60 $\mathrm{m}$, le temps de procéder à la dépose d'un petit équidé (5761-A-1). Le squelette en connexion, sans doute celui d'un petit âne, orienté nord-sud, tête vers l'est reposait sur son flanc gauche, les pattes repliées ${ }^{25}$. Des restes blanchâtres pourraient indiquer la présence lors de son enfouissement d'une natte qui aurait enveloppé et aidé à déposer le corps de l'équidé. Sa position dans la fosse marque sans

${ }^{20}$ D'un poids de 140 gr chacun (5851-M-7 et 5851-M-2).

${ }^{21}$ Ils sont les fossiles directeurs de la phase I de la Khabur Ware. Voir Baccelli \& Manuelli 2008, pl. 3 et Kolinski 2014, fig. 3.

${ }^{22}$ Pot 5851-TC-4, d'un diamètre de $17 \mathrm{~cm}$ et d'une hauteur de $5,6 \mathrm{~cm}$.

${ }^{23}$ Le déclenchement de la guerre civile syrienne peu après la campagne de 2010 n'a pas permis de faire analyser les os par un archéozoologue.

${ }^{24}$ Notamment un gros tesson provenant d'une jarre khabour ornée de 4 bandes peintes se retrouva plaqué contre l'angle nord-est extérieur du coffrage de la tombe (5851-TC-1).

${ }^{25}$ Les tarses et métatarses des membres inférieurs ainsi qu'une partie de la colonne vertébrale manquaient. Cela peut résulter d'un mauvais état de conservation du squelette de l'animal ou des résultats d'une pratique sacrificielle préférentielle (cf. Wygnanska 2017, fig. 5). 
équivoque son association avec l'inhumation de la tombe 5851. La dépouille de l'animal fut ensuite recouverte par la terre du remplissage de la fosse sur une épaisseur de $1 \mathrm{~m}$. On ne sait rien de l'éventuelle superstructure à ce dispositif funéraire. Après un laps de temps, la fosse fut recouverte par la construction de M.4292 puis de M.4885 du niveau 6b-6 (période OJZ 2).

Par son mode constructif, la composition de son matériel funéraire et l'agencement des différents éléments qui la composent, la tombe 5851 est une tombe exceptionnelle, un exemple jusqu'alors inédit.

Elle témoigne à la fois d'influence de l'oust et de l'adoption de traits culturels locaux. Son matériel céramique (coupelle et jarre peinte et tessons) indique par sa composition et le style de son décor peint une datation de la phase de pleine production de la céramique khabour ${ }^{26}$, la production céramique régionale ${ }^{27}$ durant la période OJZ 2 (ca. 18501700 av. J.-C.). Par contre, les bols en bronze et les armes, notamment la hache fenestrée, évoquent une datation de la période OJZ 1 (ca. 2000ca. 1850 av. J.-C.) un peu antérieure. La part importante des armes composant le dépôt funéraire place sans équivoque possible la tombe de la butte B de Tell Mohammed Diyab dans la catégorie des « tombes de guerriers ». Il s'agit d'une pratique funéraire relativement homogène tant du point de vue chronologique, avec la plupart de ses attestions datées entre 2000-1800 av. J.-C., que du point de vue géographique, avec une localisation principalement au Levant mais avec quelques exemplaires attestés en Mésopotamie jusque dans la péninsule arabique $^{28}$.

${ }^{26}$ La phase « céramique Khabour 2 » (= CKH 2) constitue la phase de pleine production de cette céramique, aussi nommée Early Khabur Ware ou Old Babylonian Khabur Ware. Voir Nicolle 2014a pour une présentation plus détaillée de la périodisation régionale, de la céramique et des rythmes de peuplement associés.

${ }^{27}$ L'aire de diffusion de cette céramique s'étend d'ouest en est du Khabour au grand Zab. Pour une carte de sa diffusion, voir Nicolle 2014a, fig. 1.

${ }^{28}$ Pour un rappel des différentes attestations connues des tombes de guerriers : Oren 1971, Philip 1995 et Garfinkel 2001. 


\section{LA TRADITION DES TOMBES DE GUERRIERS}

L'examen des tombes qui compose la catégorie des tombes de guerriers permet de constater la similitude des exemples et la régularité des déposes. L'archétype d'une tombe de guerrier comprend, outre l'inhumation primaire du défunt, une hache, un poignard et une ou deux lances. Une jarre, une assiette et des offrandes alimentaires principalement carnées viennent généralement compléter le dépôt. Les tombes de guerriers contiennent des armes. Si les tombes de guerriers sont caractérisées par la présence d'armes, elles ne doivent pas être confondues avec les tombes à armes. Ces dernières, beaucoup plus fréquentes chronologiquement et géographiquement, ne contiennent qu'une seule arme. Il s'agit le plus souvent d'un poignard, d'une hache ou d'une lance ${ }^{29}$.

Des tombes de ce type sont connues dès le Bronze moyen I au Levant Sud avec une période d'utilisation importante au BM IIA, que cela soit dans la vallée du Jourdain (Beth Shan, Tel Achov), dans la région du lac de Tibériade (Gesher), dans la vallée de Jezreel (Megiddo), dans la plaine côtière du Levant Nord (Sin el-Fel, Byblos, Tell Arqa, Amrit et Ras Shamra). D'autres exemples sont aussi connus en Syrie intérieure à Hama ou Tell et-Tin dans la région du lac de Homs. Ce mode d'inhumation se répand à partir du Bronze moyen II $\mathrm{B} / \mathrm{C}$ en Mésopotamie, à Chypre, jusqu'à la région est du delta nilotique (Tell el-Dab'a ou Tell el-Maskhuta) ${ }^{30}$. Là, le phénomène est lié à l'installation de populations Hyksos. En Mésopotamie, les occurrences sont nettement moins nombreuses. L'exemple offert par les tombes sous tumulus de la nécropole de Baghouz $^{31}$, sur le Moyen-Euphrate syrien, demeure isolé. Plus à l'est, quelques exemples épars sont signalés dans la vallée du Hamrin ${ }^{32}$ et dans la plaine de Rania ${ }^{33}$. Des découvertes isolées d'armes dans des contextes incertains signalent l'existence probable d'autres tombes comme à Assur et $\mathrm{Kish}^{34}$.

Au-delà des variations et des évolutions typologiques des armes, de leur composition métallurgique et du matériel céramique associé, le

${ }^{29}$ Gernez 2014, p. 48.

30 Philip 1995.

${ }^{31}$ Du Mesnil du Buisson 1948.

32 À Tell Yelkhi niveau V daté de la période Isin Larsa, Bergamini 1984, p. $235-236$.

33 À Tell Shemshara dans un niveau daté du règne de Samsi-Addu, Læssoe 1963, pl. 12(b).

34 Voir Philip 1995. 
principe général qui organise ces tombes signale l'existence d'une pratique commune, issue d'une tradition levantine qui se cristallise durant le dernier tiers du $\mathrm{III}^{\mathrm{e}}$ millénaire. Elle se diffuse ensuite du delta du Nil à la Mésopotamie avant de se dissoudre progressivement dans les sphères culturelles locales, pour disparaître complètement à la fin du Bronze moyen $\mathrm{II}^{35}$.

Des interrogations subsistent quant à la valeur à accorder à de telles tombes. S'agissait-il réellement des tombes des membres d'une élite guerrière ? Si les données anthropologiques font souvent défaut, quelques éléments disponibles indiquent que l'on a affaire exclusivement à des inhumations de sexe masculin. Les pourcentages obtenus sur quelques nécropoles levantines du Bronze moyen les mieux conservées et les plus fouillées indiquent qu'environ un homme sur deux était ainsi inhumé ${ }^{36}$. Même pour les sociétés levantines fortement militarisées de l'âge du Bronze, un tel rapport est trop élevé pour qu'il puisse signaler des individus appartenant à une élite guerrière. De plus, une étude comparative entre les armes trouvées dans ces tombes et celles représentées dans diverses scènes guerrières de Mésopotamie permet de constater qu'elles n'appartiennent pas au même arsenal guerrier. Dans les scènes de guerre, elles se composent de lances, d'arcs, de boucliers et de casques ${ }^{37}$. Dans les tombes de guerriers, haches, lances et surtout poignards constituent le matériel habituel. Les variantes de l'armement déposé dans les tombes permettent de distinguer différentes valeurs des individus. Il y a d'un côté les armes de soldats et de l'autre des armes, parfois d'apparat, dont la fonction est une valeur essentiellement symbolique et qui sont destinées à souligner la masculinité de certains défunts à travers la valorisation d'un statut idéalisé du guerrier. Ce sont des soldats que l'on retrouve dans certaines tombes royales d'Ur (notamment la tombe royale PG 780) et qui font office de morts d'accompagnement ${ }^{38}$. Ce sont des individus distingués pour certaines valeurs que l'on retrouve dans des inhumations primaires simples dans les tombes de guerriers.

35 Notons qu'au Dynastique archaïque III, l'inhumation de certains individus associés à plusieurs armes fut déjà pratiquée. La vague des tombes de guerriers de la fin du III ${ }^{\mathrm{e}}$ millénaire et du Bronze moyen n'a aucune filiation avec ce précédent.

36 Garfinkel 2001, p. 155. Un constat fait dans les nécropoles de Gezer et Rehov où selon le comptage, la moitié des inhumations sont le fait de femmes et un homme sur deux est inhumé dans une tombe de guerrier. Le fait d'inhumer la moitié des hommes avec des armes signale par conséquent qu'il ne peut s'agir des représentants d'une élite guerrière.

37 Rehm 2003, p. 138.

38 Sur la notion de mort d'accompagnement : Testart 2004. 
Les tombes de guerriers ne sont pas les inhumations des membres d'une élite guerrière ou d'une classe sociale de guerriers. Plus certainement elles permettent, grâce à la charge symbolique des armes associées, de caractériser le rang social de certains adultes de sexe masculin. Il s'agit alors de «tombes de rangs » et non pas de «tombes de guerriers ». D'ailleurs, la disparition progressive d'une telle pratique funéraire ne doit pas être mise en relation avec une diminution de la place accordée dans la société aux activités militaires. Au Levant comme en Mésopotamie, le Bronze moyen II n'est en rien plus pacifique que la période précédente ${ }^{39}$. Dans la représentation sociale des morts, en tout cas en Djézireh, elle correspond à la fin de la prééminence de certains individus au profit d'une famille ou d'un groupe social comme le marque le développement à partir de ce moment des caveaux à inhumations multiples.

La diffusion en Mésopotamie au Bronze moyen du modèle de la tombe de guerrier était soupçonnée depuis longtemps, avant même la découverte de l'une de ces tombes, par des découvertes isolées d'armes faites à Kish, Assur et Tell Shemshara et d'autres achetées par des musées, sans indication de provenance ${ }^{40}$.

La zone qui documente le mieux cette pratique funéraire est la vallée du Hamrin. Des fouilles de sauvetage italiennes ont permis la découverte de plusieurs tombes de guerriers à Tell Yelkhi. Certaines d'entre elles pourraient dater de la fin du $\mathrm{III}^{\mathrm{e}}$ millénaire ${ }^{41}$. La concentration de ces tombes autour d'un bâtiment administratif de l'époque tend à signaler le haut statut du groupe. Dans d'autres nécropoles de la même période (Tell Songor, Tell Hassan ou Tell Abu Husseini) quelques armes sont signalées en faible nombre dans les tombes ${ }^{42}$. Des rapports préliminaires succincts rendent difficile de déterminer s'il s'agissait de tombes à armes ou de tombes de guerriers. Dans la même région, les fouilles non publiées de Tell el-Seib ont produit d'autres exemples de ce type de tombes du début du II ${ }^{\mathrm{e}}$ millénaire ${ }^{43}$. L'une d'entre elles était associée à l'inhumation d'un équidé, sans doute un âne ${ }^{44}$. Les autres attestations de tombes de guerriers en Mésopotamie sont rares. Une est mentionnée à Tell

${ }^{39}$ Cohen 2012, p. 312.

40 Philip 1995, p. 146.

${ }^{41}$ Fiorina 1985 , p. $62-63$.

${ }^{42}$ Fiorina 1984, fig. 27 ; 1985, p. 63.

43 Pour de courts rapports préliminaires, voir Postgate \& Watson 1979; Roaf \& Postgate 1981.

${ }^{44}$ Hannoun 1984, il s'agit de la tombe 9. 
Shemshara dans la plaine de Rania ${ }^{45}$. Dans le triangle du Khabour à Tell Chagar Bazar, une autre contenait une hache similaire à celles découvertes sur plusieurs sites de la vallée du Hamrin, ainsi qu'un poignard et une pointe de lance ${ }^{46}$. D'autres ne contenaient qu'une $\operatorname{arme}^{47}$. Les contextes de fouille, de conservation et de publication ne permettent pas d'être très précis. Il pourrait s'agir de tombes à armes.

Pour la région du triangle du Khabour et l'exemple de Tell Mohammed Diyab, l'élément de comparaison le plus proche géographiquement est celui offert par la nécropole de Baghouz sur le moyen Euphrate syrien où onze tombes de guerriers ont été découvertes sur le mamelon II, constituant $23 \%$ des tombes identifiées ${ }^{48}$.

Malgré des différences dans la composition des tombes (parois en briques crues dans une fosse à Tell Mohammed Diyab, sous tumuli à Baghouz), certaines régularités dans le traitement du corps confirment l'appartenance à une même tradition funéraire composée d'inhumations primaires individuelles. Le mort est placé sur le côté, les jambes pliées. Excepté la présence de mobiliers en bois à Baghouz composés de lits et de tables d'offrande, le reste du matériel funéraire est assez similaire. Il s'agit de haches placées le plus souvent au niveau ou en arrière de la tête et des pointes de lance placées au pied du corps et plus rarement au niveau de la tête. Ces objets constituent la norme dans les armes. Les couteaux sont plus rares. Un seul exemplaire a été retrouvé dans une tombe de Baghouz (tombe Z-122). Les bols, parfois en bois le plus souvent en céramique, constituent une autre dépose habituelle. La découverte de deux bols en bronze dans la tombe 5851 de Tell Mohamed Diyab représente une situation inédite. Jarre ou cruche constituent le dernier élément récurrent du matériel d'une tombe de guerrier.

\section{Les tombes D’ÉQuidés Au PROCHE-ORIENT}

L'inhumation du «guerrier» dans la tombe 5851 sur la butte B était associée à celle d'un équidé dont le squelette en connexion a été retrouvé dans la fosse à environ $1 \mathrm{~m}$ sous la surface. L'animal, sans doute un petit

45 Laessoe 1963, pl. 12 b et p. 113.

46 Mallowan 1937, fig. 8.

47 Mallowan 1947, p. 85-86.

48 du Mesnil du Buisson 1948. Sur un corpus de 185 tombes du Bronze moyen plus 47 réutilisées. 25 contenaient des armes soit 10\% du total (Philip 1995). 
âne, fut probablement tué à proximité et son corps déposé dans la fosse en cours de comblement.

Il vient enrichir la liste des tombes d'équidés qui apparaissent dès le milieu du III $^{\mathrm{e}}$ millénaire en Irak et en Syrie intérieure. Leur nombre connaît une nette augmentation au $\mathrm{II}^{\mathrm{e}}$ millénaire. Différents contextes d'enfouissement sont documentés. Il y a des tombes isolées, des tombes découvertes sous des murs, à proximité immédiate ou dans des édifices religieux. L'essentiel de ces tombes se trouve en Syrie-Palestine et en Égypte. Elles peuvent parfois être associées à des tombes humaines. Les squelettes des équidés sont alors retrouvés devant l'entrée, à l'intérieur, en dehors ou sur la tombe.

Quels que soient les contextes d'enfouissement, à l'évidence, ces tombes d'équidés signalent que pour le monde des vivants ${ }^{49}$ comme pour le monde des morts ${ }^{50}$, ces animaux avaient une valeur et un prestige qui leurs conféraient un statut particulier et une forte charge symbolique ${ }^{51}$. Les textes viennent confirmer la valeur de ces animaux. Ils y sont souvent associés à des élites ${ }^{52}$. Ils renseignent aussi sur les différents modes d'utilisation de ces animaux dans des rituels qui ne sont pas exclusifs, principalement des sacrifices. Les équidés peuvent être immolés en l'honneur d'un dieu $^{53}$, lors de pacte de sang entre groupes, de prestations de serments ou de traités ${ }^{54}$. Un tel rôle explique la présence de tombes

49 Pour une description du rôle des équidés et de leur valeur socio-économique au Bronze moyen, cf. Michel 2004.

${ }^{50}$ Pour des descriptifs de ces attestations : Zarins 1986, Greenfield et al. 2012 et Wygnanska 2012.

${ }^{51}$ L'âne, la mule et le mulet n'ont alors pas les valeurs péjoratives (entêtement et stupidité) qu'ils ont de nos jours ; Greenfield et al. 2102, p. 45.

${ }^{52}$ Scurlock 2002, p. 392-393. En outre, l'association entre équidés et l'aristo-cratie amorrite transparait clairement dans plusieurs textes des archives de Mari. Par exemple Bahdî-Lîm, gouverneur de Mari écrit au roi Zimri-Lîm pour lui rappeler que pour son retour et son entrée triomphale à Mari (en ZL 4), il ne doit pas monter de chevaux, animaux du Nord. Il doit être sur un nûbalum (une sorte de litière de transport) tiré par des mules; Durand 1998, p. 484-488. Chevaucher une mule serait plutôt à associer à une symbolique akkadienne; Lafont 2000, p. 214. Il y aurait deux modes royaux de parade à mettre en relation pour le premier avec les régions occidentales et la sphère culturelle amorrite et pour la seconde avec le monde de l'ouest, de la Mésopotamie et les populations akkadiennes.

${ }^{53}$ Des textes ougaritiques mentionnent des sacrifices d'âne fait en l'honneur de Baal ; Way 2010, p. 214.

54 Les animaux souvent mentionnés dans ces sacrifices liés à des rituels d'alliance sont des bœufs, des chèvres, des chiots et des ânons. Voir plusieurs attestations extraites des archives mariotes dans Durand 1997, p. 443-446 ; Charpin 1993, p. 184-186. Le sacrifice d'ânon (ânon d'alliance) est aussi attesté dans le cas d'alliances internationales entre souverains ; Jacquet 2004, p. 44. 
d'équidés dans les temples. Ils apparaissent aussi dans des rites de fondation comme le signalent des découvertes de tombes d'équidés sous des murs ou des temples ${ }^{55}$. Pour établir et comprendre les différentes fonctions et valeurs que prennent ces animaux et qui varient apparemment selon les contextes sacrificiels, il faudrait pouvoir prendre en compte la variété des races (âne, mule, mulet, onagre, chevaux sauvages ou domestiques). Ce type d'étude est malheureusement encore peu développé.

Dans les cas moins fréquents d'association de tombes d'équidés à des rites funéraires, les contextes sont tout aussi variés ${ }^{56}$ et les quelques exemples connus diffusés sur une vaste aire géographique. Au Levant, les exemples les plus anciens datent du Bronze ancien $\mathrm{I}^{57}$. La pratique est surtout attestée au Bronze ancien III $^{58}$ et au Bronze moyen II $^{59}$ jusque dans le delta du Nil ${ }^{60}$. Toutefois, les exemples connus sont encore peu nombreux et souvent fragmentaires. Le cas illustré par la nécropole d'Umm el-Mara ${ }^{61}$ dans la plaine du Jabbul reste exceptionnel ${ }^{62}$. À la suite d'une reformulation des rites funéraires au tournant des $\mathrm{III}^{\mathrm{e}} / \mathrm{II}^{\mathrm{e}}$ millénaires, les sacrifices suivis de leurs inhumations d'équidés deviennent plus fréquents. C'est le cas au Bronze moyen sur toute la façade levantine. Le développement et la diffusion de cette pratique d'immolation d'équidés sont souvent associés aux mouvements de populations amorrites, et dans le delta est du Nil, à l'installation de groupes de Hyksos. La

55 Par exemple, dans une fosse associée au temple forteresse in antis de Tel Haror dans le Levant Sud au Bronze Moyen ; Oren 1997, p. 265-266. Il peut aussi arriver qu'il s'agisse d'une mort naturelle et que l'on se soit débarrassé du corps de l'animal. Le cas est attesté à Tell Mohammed Diyab où le squelette d'un âne a été découvert dans une fosse d'époque médio-assyrienne ; Castel 1992, p. 41.

56 La pratique est attestée sur différents sites du Levant, de Mésopotamie, d'Égypte et de la péninsule arabique dès le III ${ }^{\mathrm{e}}$ millénaire ; Greenfield et al. 2012, p. 26.

57 À Lachish ; Tufnell 1958.

58 Par exemple à Tell es-Sakan (Miroschedji et al. 2001), Tell es-Safi (Greenfield et al. 2012).

59 Tell el-Jemmeh (Wapnish 1997), Tell Haror (Bar-Oz et al. 2013), Jericho (Kenyon 1960, p. 306-308).

60 Principalement Tell el-Dab'a (Bietak 1997, p. 103) et Tell el-Makshuta (Holladay 1997).

61 Nichols \& Weber 2006. Des inhumations d'équidés associés à des tombes mais aussi dans des structures architecturales particulières ont été retrouvées à Umm el-Mara dans la nécropole de son élite implantée au cœur de la ville.

62 On ne peut guère mentionner que les découvertes dans des niveaux du $\mathrm{III}^{\mathrm{e}}$ millénaire de restes d'équidés associés à des êtres humains ont été faites sur le Moyen Euphrate voisin dans le White Monument de Tell Banat (Porter 2002) et à Halawa (Orthmann 1981) dans des structures datées du Bronze ancien IV. 
pratique reste encore peu documentée en Mésopotamie ${ }^{63}$, peut-être du fait de son accroche essentiellement occidentale. Seulement six exemples sont connus en Mésopotamie, dans la vallée du Hamrin ${ }^{64}$, dans le bassin de Aditha ${ }^{65}$, en Mésopotamie centrale ${ }^{66}$ et dans la Djézireh syrienne ${ }^{67}$.

Les contextes d'utilisation des équidés dans les rites funéraires sont de plusieurs ordres. Ils peuvent être le résultat d'une offrande sacrificielle consommable ou non, ou bien être assimilés à un élément du mobilier funéraire.

L'équidé peut être considéré comme une ressource carnée, associée à des festins en l'honneur du défunt ou pour nourrir celui-ci dans l'audelà. Une telle interprétation prévaut quand la carcasse de l'animal est incomplète ou qu'elle porte sur ses os des traces de boucherie ${ }^{68}$. Les équidés sont parfois sacrifiés et enterrés seuls ou avec d'autres animaux. Il s'agit le plus souvent de canidés et de caprinés comme dans un exemple datant de 2250 av. J.-C. de Tell Brak ou un autre du Bronze moyen découvert à Tell Arbid ${ }^{69}$.

S'il est utilisé comme un mobilier funéraire, on le trouve parmi d'autres biens pouvant servir pour la vie après la mort du défunt tout en ayant été un animal indispensable à sa vie quotidienne, au même titre que la nourriture, les habits, les chars ou des serviteurs ${ }^{70}$.

${ }^{63}$ De plus, les ossements des équidés sont souvent fragmentaires et retrouvés désarticulés.

64 À Tell Madhhur, Abu Salabikh, Tell Razuk, Tell Abu Qasim, Tell Ababra, cf. Zarins 1986 pour une présentation générale ; et à Tell es-Seib (Hannoun 1984, p. 70-71).

65 À Tell Ababra (tell E) ; Piesl-Trenkwalder 1981-1982, p. 252.

66 À Tell ed-Der; Gasche 1989, p. 53.

67 À Tell Arbid, Piatkowska-Malecka \& Wygnańska 2012, p. 71, 74 et Wygnańska 2017, p. 151 signalent d'après une communication personnelle de R. Kolinski le cas d'un squelette d'âne déposé sur la couverture de la tombe à ciste (G1/P/37/63-2010). Sur le même site, un autre cas est attesté dans la tombe à puits G7/37/62 (Kolinski 2009) ainsi que dans le puits d'accès d'un caveau contenant les squelettes de quatre humains (G8/ G9-S-37/55-2001). À Tell Mozan, une autre dépose de carcasse complète d'une ânesse est attestée à l'entrée d'une tombe familiale (tombe 37) ; Dohmann-Pfälzner \& Pfälzner 2001, p. 129-133. On peut maintenant y ajouter le cas de la tombe 5854 de Tell Mohammed Diyab.

${ }^{68}$ C'est le cas à Umm el-Mara (tombes 3 et 4), Schwartz et al. 2006, p. 610-614. D'autres exemples sont attestés particulièrement au Levant comme à Jéricho où, dans une tombe du Bronze moyen I, la moitié du squelette d'un équidé a été retrouvée ; Kenyon 1960, p. 306-309.

${ }_{69}$ Tell Brak: Oates et al. 2001, p. 41-50 ; Tell Arbid: Piątkowska-Małecka \& Wygnańska 2010.

${ }_{70}$ Des équidés associés à des chars ont été retrouvés dans certaines tombes comme à Kish ou Ur. Il s'agit d'animaux d'attelage ou de transport pour accompagner le défunt dans l'au-delà. D'autres exemples dans des tombes moins monumentales sont signalés à Umm el-Marra, Tell Abadra, Tell Halawa, etc. 
Une autre interprétation est envisageable dans le cas de squelette complet de l'animal. Il pourrait s'agir d'animaux ayant servi à conduire le corps du défunt vers sa dernière demeure ou une sorte « d'animaux favoris » liés à la fonction ou au métier qu'exerçait le défunt. Dans le cadre des découvertes faites dans le delta nilotique, il a été émis l'hypothèse qu'il pouvait signaler l'implication du défunt dans les caravanes notoirement associées dans la région à des expéditions minières. Le défunt associé à la dépouille de l'âne aurait été un de ces chefs caravaniers ${ }^{71}$. La dépose de l'âne signalerait l'appropriation par les membres d'une élite de pratiques ou d'images associées au commandement dans le contexte d'expéditions minières dans le cadre égyptien et sud levantin. En Mésopotamie du Nord, cette forme d'appropriation peut concerner le monde des caravanes de marchands. Les usages n'étant pas exclusifs, on peut envisager conjointement une fonction plus religieuse ou une valeur plus symbolique au sacrifice de l'animal. Dans tous les cas, la dépose d'une carcasse complète ou en connexion d'un équidé et son association avec une tombe humaine est un rite funéraire particulier qui doit être considéré comme un marqueur de prestige $\mathrm{e}^{72}$.

À l'évidence, l'enterrement, après immolation ou non, d'un équidé (le plus souvent âne et onagre, plus rarement le cheval) était une pratique répandue à l'ensemble de la Mésopotamie et du Levant. La concentration des exemples connus montre que sa pratique relevait principalement de la sphère culturelle ouest-sémitique du Levant et du delta nilotique. Bien qu'elle ait été le fait de populations ou d'ethnies variées (Sumériens, Amorrites, Cananéens, Hyksos, Hourrites), elle ne constituait pas, notamment au Bronze moyen, un rituel funéraire commun. L'âne ou la mule ont dans le monde amorrite de cette époque une valeur symbolique manifeste qui les associe aux personnages de haute naissance. Une telle caractérisation explique le nombre peu élevé de sacrifices connus d'équidés accompagnant des inhumations humaines. Les attestations de tels rituels funéraires associés avec des tombes de guerriers sont encore plus rares. Quelques cas sont connus dans le delta nilotique ${ }^{73}$, au Levant $\mathrm{Sud}^{74}$, sur

${ }^{71}$ L'hypothèse a été développée par M. Bietak (1997), et reprise notamment par G. Philip (2006, p. 238).

72 Schwartz et al. 2006, p. 630-634 ; Vila 2006, p. 117-120 ; Weber 2008, p. 500-514.

${ }^{73}$ Comme à Tell el-Maskhouta ; Holladay 1997, p. 195, 223.

${ }^{74}$ Exemple à Jéricho, sur quatre inhumations associées à des équidés signalées (Kenyon 1960, p. 306-308), une seule entre dans la catégorie des tombes de guerriers. 
le Moyen-Euphrate ${ }^{75}$. En Mésopotamie, si le défunt est enterré avec ses armes comme dans une tombe de guerrier, la position des équidés souvent par paire et côte à côte indique qu'il s'agit d'une autre catégorie de tombes, celle des tombes à $\operatorname{char}^{76}$.

\section{Aux origines D’une Culture RÉGionale}

La tombe 5851 de Tell Mohammed Diyab est un exemple typique de tombe de guerrier, une pratique du début du $\mathrm{II}^{\mathrm{e}}$ millénaire qui disparaît vers 1800 av. J.-C. La typologie de son armement témoigne de fortes connexions avec la nécropole de Baghouz sur le moyen Euphrate et d'autres nécropoles du Levant Nord et du Levant Sud du début du Bronze moyen. Son association exceptionnelle avec l'immolation d'un petit équidé signale son insertion dans le monde amorrite où la pratique est fréquente. Le personnage inhumé était un personnage important. Sa richesse est signalée par les objets en bronze qui constituent son matériel funéraire. Ils sont plus abondants que dans la plupart des autres tombes de guerriers. Sa dignité est révélée par l'immolation d'un ânon, un animal alors associé aux personnes de haut rang et aux politiques importants. L'âne peut aussi être considéré comme « un animal favori » lié à la fonction du défunt. On peut donc proposer l'hypothèse qu'il s'agissait d'un marchand ou d'un chef caravanier d'une des nombreuses caravanes de marchands en route pour Kültepe ou de retour vers Assur ${ }^{77}$. Décédé durant l'un de ces voyages il aurait été enterré lors d'une étape à Tell Mohammed Diyab ${ }^{78}$ dans le secteur politico-économique du site

75 À Halawa, trois équidés étaient associés à une tombe de guerrier dans laquelle se trouvait aussi une jeune femme.

${ }^{76}$ Il en est ainsi dans le bassin du Hamrin à Tell Madhur. une grande tombe à char (tranchée 5G) datée de 2300-2200 av. J.-C. contenant le squelette d'un homme avec ses armes, associé à deux squelettes d'âne et les restes de bois de ce qui pourraient être les vestiges d'un char, similaire à ceux de Kish et d'Ur ; Killick \& Roaf 1979. Un cas de figure similaire se retrouve à Tell Razuk (Uch Tepe) dans une tombe de la période akkadienne (vers 2300 av. J.-C). Une grande tombe dite de guerriers contenant dans un loculus un squelette humain et ses armes avec dans la grande chambre le reste du mobilier funéraire et les squelettes de deux ânes disposés en attelage côte à côte (Gibson et al. 1981, p. 73-74). Une telle composition place de fait la tombe dans la catégorie des tombes à char.

77 Pour une description des différentes personnes composant une de ces caravanes : Michel 1992.

78 Tell Mohammed Diyab est sur l'une des principales routes reliant à cette époque Assur, dans la zone de diffusion de la céramique khabour et Kültepe/Kaneš (Kolinski 2014 , p. 14 et fig. 1). Une proposition d'identification de ce site avec la ville d'Apum a été faite ; Eidem 2008. Le nom ne se référait pas encore à un pays situé autour de 
alors abandonné ${ }^{79}$. La mémoire de son lieu de sépulture ne perdura pas. Il fut rapidement recouvert lors de l'installation d'une nouvelle population et de la construction de nouveaux entrepôts à la phase suivante.

La pratique d'inhumation des «tombes de guerriers », en fait des tombes de rangs, relève d'un phénomène de représentation social. L'expression du pouvoir du défunt est liée à un prestige personnel exprimé dans une référence à un idéal du guerrier. L'usage est généralisé au Levant Sud, au Levant Nord et à la Mésopotamie depuis le Bronze ancien IV jusqu'à la fin du Bronze moyen. La pratique du sacrifice d'équidés et la charge symbolique accordée aux ânes sont souvent associées au monde culturel amorrite. L'importance accordée à cette espèce animale dépasse cependant largement la sphère d'une éventuelle "koiné amorrite » puisqu'elle est attestée du $\mathrm{III}^{\mathrm{e}}$ millénaire au $\mathrm{I}^{\mathrm{er}}$ millénaire et parfois associée à d'autres ethnies linguistiques comme celle des Hourrites ${ }^{80}$. Il ne s'agit pas d'une pratique exclusivement amorrite.

Tout comme ces pratiques, certains éléments de la culture matérielle, comme l'armement, relèvent d'une identité globalisante. Leur distribution révèle un réseau de distribution supra régional irriguant une grande partie du Proche-Orient. Cependant, des régionalismes sont perceptibles dans l'adoption de certains types d'arme. Ils deviennent évidents dans la constitution d'une culture céramique comme celle de la céramique khabour.

Il est difficile et peu signifiant de déterminer grâce à sa culture matérielle l'appartenance du mort à l'ethnie amorrite avant tout identifiée comme une communauté linguistique, de plus difficile à cerner ${ }^{81}$. Dans le même ordre d'idée, la multiplicité des origines perceptibles dans les différents éléments qui constituent la tombe témoigne d'un pluralisme culturel à l'œuvre dans la région en ce début de la période OJZ 2.

Shubat-Enlil. Apum est effectivement mentionnée comme une étape dotée d'un karum dans les textes de Kültepe II (contemporaine de la phase OJZ 1) dans un itinéraire reliant Hahhum à Kaneš. Un sceau-cylindre découvert à Tell Mohammed Diyab de style cappadocien et daté du XIX ${ }^{\mathrm{e}}$ siècle a été considéré comme la preuve de ce rôle d'étape pour des caravanes de marchands (Castel 1990, p. 53 et 1992, p. 47). Toutefois, d'autres candidats pour la ville d'Apum sont possibles comme Tell Aid (Eidem 2008, p. 269-270) et l'équivalence avec une autre ville Azamhul a été proposée dès les premières campagnes de fouilles à Tell Mohammed Diyab avec autant d'arguments convaincants (cf. Charpin 1990).

79 Tell Mohammed Diyab a connu durant la période amorrite plusieurs phases de retrait ou d'abandon. Ces discontinuités caractérisent l'occupation de la région, cf. Nicolle 2014b.

${ }^{80}$ Way 2010, Silver 2014.

81 Durand 2012. 
Il illustre la variété des flux migratoires en œuvre ${ }^{82}$ en Djézireh comme ailleurs $^{83}$. La tombe 5851 doit être considérée pour ce qu'elle est : le témoignage de l'association d'éléments culturels aux origines variées qui va constituer le creuset d'une nouvelle culture régionale dans une région en cours de réoccupation en ce début de $\mathrm{II}^{\mathrm{e}}$ millénaire.

\section{BIBLIOGRAPHIE}

Andrae W., 1922 : Die Archaischen Ischtar-Tempel in Assur, Wissenschaftliche Veröffentlichung der Deutschen Orient-Gesellschaft 39, Osnabrück.

Baccelli G. \& F. Manuelli, 2008 : «Middle Bronze Khabur Ware from Tell Barri / Kahat », dans J. M. Córdoba et al. (éd.), Proceedings of the $5^{\text {th }}$ International Congress on the Archaeology of the Ancient Near East, Madrid, April 3-8 2006. Volume I, Madrid, p. 187-205.

Bar-Oz G., P. Nashoni, H. Motro \& E. Oren, 2013 : « Symbolic Metal Bit and Saddlebag Fastenings in a Middle Bronze Age Donkey Burial », PLOS ONE 8(3), DOI : 10.1371/journal.pone.0058648.

Bergamini G., 1984 : «The Excavations in Tell Yelkhi », Sumer 40, p. 224-244. Bietak M., 1997 : « The Center of the Hyksos Rule: Avaris (Tell el-Dab'a)», dans E. D. Oren (éd.), The Hyksos: New Historical and Archaeological Perspectives, University Museum Monograph of Pennsylvania 96, Philadelphie, p. 87-139.

Buccellati G., 1992 : « Ebla and the Amorites », Eblaitica 3, p. 83-104.

Castel C., 1990 : «Découverte d'un sceau-cylindre paléo-assyrien : Mohammed Diyab, une étape pour les marchands assyriens en route vers la Cappadoce », dans J.-M. Durand (éd.), Tell Mohammed Diyab, campagnes 1987 et 1988, Cahiers de NABU 1, Paris, p. 51-58.

1992 : «Explorations dans la ville basse de Mohammed Diyab », dans J.-M. Durand (éd.), Recherches en Haute Mésopotamie, Tell Mohammed Diyab, campagnes 1990 et 1991, Mémoires de NABU 2, Paris, p. 39-54.

Charpin D. \& N. Ziegler, 2003 : Mari et le Proche-Orient à l'époque amorrite, Essai d'histoire politique, FM V, Mémoires de NABU 6, Paris.

Charpin D. 1990 : «Conclusions et perspectives : Tell Mohammed Diyab, une ville du pays d'Apum », dans J.-M. Durand (éd.), Tell Mohammed Diyab, campagnes 1987 et 1988, Cahiers de NABU 1, Paris, p. 117-122.

${ }^{82}$ Rappelons l'hypothèse de la toponymie en miroir proposée par D. Charpin avec des dominations doubles qui seraient le souvenir de la région d'origine de certaines populations. Ainsi, un pays d'Apum est connu dans la région de Damas et un autre dans la région de Tell Leilan ; Charpin 2003, p. 10-12 et 16. Pour autant, la superposition des nombreux mouvements migratoires à cette période empêche d'établir des relations simples entre une population et un lieu d'origine supposé. Trois de ces grandes vagues migratoires ont été identifiées par D. Charpin et N. Ziegler 2003, p. 29-30 ; une première vague migratoire à la fin de la III $^{\text {e }}$ Dynastie d'Ur, une seconde vers 1900 av. J.-C. et une troisième vers 1830 av. J.-C.

${ }^{83}$ Un phénomène similaire se constate dans la vallée du Hamrin. 
1993 : «Un souverain éphémère en Ida-Maras : Išme-Addu d'Ašnakkum », MARI 7, p. 165-191.

2003 : « La "toponymie en miroir" dans le Proche-Orient amorrite », RA 97, p. 3-33.

Cohen S. L., 2012 : «Weaponry and Warrior Burials: Patterns of Disposal and Social Change in the Southern Levant », dans R. Matthews \& J. Curtis (éd.), Proceedings of the $7^{\text {th }}$ ICAANE 12 April - 16 April 2010, British Museum and UCL, London. Volume 1: Mega-cities \& Mega-sites. The Archaeology of Consumption \& Disposal. Landscape, Transport \& Communication, Wiesbaden, p. 307-319.

Dohmann-Pfälzner H. \& P. Pfälzner, 2001 : « Ausgrabungen der Deutschen Orient-Gesellschaft in der zentralen Oberstadt von Tall Mozan/Urkes. Bericht über die in Kooperation mit dem IIMAS durchgeführte Kampagne $2000 », M D O G$ 133, p. 97-139.

Durand J.-M., 1998 : Les documents épistolaires du palais de Mari, Tome II, LAPO 17, Paris.

2012 : «Réflexions sur un fantôme linguistique », dans C. Mittermayer \& S. Ecklin (éd.), Altorientalische Studien zu Ehren von Pascal Attinger, OBO 256, Fribourg, p. 165-191.

Fiorina P., 1984 : « Excavations at Tell Hassan, preliminary report », Sumer 40, p. $277-289$.

1985 : «Funeral Customs », dans E. Quarantelli (éd.), Land between Two Rivers. Twenty years of Italian Archaeology in the Middle East. The Treasures of Mesopotamia, Turin, p. 62-63.

Gasche H., 1989 : La Babylonie au 17ème siècle avant notre ère. Approche archéologique, problèmes et perspectives, Mesopotamian History and Environnement. Series II. Memoirs 1, Gand.

Garfinkel Y., 2001 : «Warrior Burial Customs in the Levant During the Early Second Millennium B.C. », dans S. R. Wolff (éd.), Studies in the Archaeology of Israel and Neighbouring Lands in Memory of Douglas L. Esse, Studies in Ancient Oriental Civilization 59, Chicago, p. 143-161.

Gernez G., 2007 : L'armement en métal au Proche-Orient et Moyen-Orient. Des origines à $1750 \mathrm{av}$. J.-C. Thèse dactylographiée de doctorat d'Archéologie présentée et soutenue publiquement le 5 décembre 2007 à l'Université de Paris 1 Panthéon-Sorbonne, Paris.

— 2014-15 : « Tombes de guerriers et tombes à armes au Liban au Bronze moyen », Archaeology \& History in the Lebanon 40-41, p. 45-74.

Greenfield H. J., I. Shai \& A. Maeir, 2012 : « Being an "ass": An Early Bronze Age burial of a donkey from Tell es-Safi/Gath, Israel », Bioarchaeology of the Near East 6, p. 21-52.

Haller A., 1954 : Die Gräber und Grüfte von Assur, WVDOG 65, Berlin.

Hannoun N., 1984 : « Excavations at Tulul Baradan, Al-Seib and Haddad », Sumer 40, p. 70-72.

Holladay J. S., 1997 : « The Eastern Nile Delta the Hyksos and Pre-Hyksos Periods: Toward a Systematic/Socioeconomic Understanding », dans E. Oren (éd.), The Hyksos: New Historical and Archaeological Perspectives, University Museum Monograph of Pennsylvania 96, Philadelphie, p. 183-252. 
Jacquet A., 2004 : «Les rituels royaux à l'époque amorrite. Religion et politique d'après les archives de Mari », Hypothèses 2004/1, p. 35-47.

Kenyon K. M., 1960 : Excavations at Jericho. Vol. I: The Tombs Excavated in 1952-4, The British School of Archaeology in Jerusalem, Londres.

Kolinski R., 2012 : " Adam Mickiewicz University excavations in Sector P at Tell Arbid (Spring 2009) », PAM 21, p. 537-557.

2014 : « $20^{\text {th }}$ Century BC in the Khabur Triangle Region and the Advent of the Old Assyrian trade with Anatolia », dans D. Bonatz (éd.), The Archaeology of Political Spaces. The Upper Mesopotamian Piedmont in the Second Millennium BCE, TOPOI - Berlin Studies of the Ancient World 12, Berlin, p. 11-34.

Laessoe J., 1963 : People of Ancient Assyria, Londres.

Lafont B., 2000 : «Cheval, âne, onagre et mule dans la haute histoire mésopotamienne, quelques données nouvelles», dans Les animaux et les hommes dans le monde syro-mésopotamien aux époques historiques, Topoi. OrientOccident. Supplément 2, p. 207-221.

Langdon S., 1924 : Excavations at Kish. Volume I, Paris.

Mallowan M. E. L., 1937 : " The Excavations at Tell Chagar Bazar and an Archaeological Survey of the Khabur Region, Second Campaign, 1936 », Iraq 4/2, p. 91-177.

- 1947 : «Excavations at Brak and Chagar Bazar », Iraq 9, p. 1-259.

Mesnil du Buisson R. du, 1948 : Baghouz, l'ancienne Corsoté, le tell archaïque et la nécropole de l'âge du Bronze, Documenta et Monumenta Orientis Antiqui 3, Leiden.

Michel C., 2004 : « The perdum-mule, a mount for distinguished persons in Mesopotamia during the first half of the second millennium BC », dans F. Barbro Santillo (éd.), PECUS. Man and animal in antiquity. Proceedings of the conference at the Swedish Institute in Rome, September 9-12, 2002, The Swedish Institute in Rome. Projects and Seminars 1, Rome, p. 190-200.

Miroschedji P. de et al., 2001 : «Les fouilles de Tell es-Sakan (Gaza) : Nouvelles données sur les contacts égypto-cananéens aux IV $\mathrm{IV}^{\mathrm{e}} \mathrm{III}^{\mathrm{e}}$ millénaires », Paléorient 27/2, p. 75-104.

Müller-Karpe M., 2004 : «Katalog I : Untersuchung Metallobjekte aus Mesopotamien », dans H. Hauptmann \& E. Pernicka (éd.), Die Metallindustrie Mesopotamiens von den Anfängen bis zum 2. Jahrtausend v. Chr., Rahden, p. 1-90.

Nichols J. J. \& J. A. Weber, 2006 : « Amorites, Onagers, and Social Reorganization in Middle Bronze Age Syria », dans G. Schwartz \& J. Nichols (éd.), After Collapse: The Regeneration of Complex Societies, Tucson, p. 38-57.

Nicolle C., 2012 : «Rapport préliminaire de la campagne de l'automne 2010 à Tell Mohammed Diyab (Djézireh syrienne) », Chronique Archéologique en Syrie VI, p. 123-139.

2014a : « Le triangle du Habur pendant la première moitié du II ${ }^{\mathrm{e}}$ millénaire av. n.è. Rythmes et nature des occupations », dans N. Ziegler \& E. CancikKirschbaum (éd.), Entre les fleuves - II. D'Assur à Mari et au-delà, BBVO 24, Gladbeck, p. 21-48.

2014b : «Une question de rythme au pays d'Apum : les quatre agglomérations de Tell Mohammed Diyab durant la période Khabour », dans 
L. Marti (éd.), La famille dans le Proche-Orient ancien : réalité, symbolismes, et images. Proceedings of the $55^{\text {th }}$ Rencontre Assyriologique Internationale at Paris, 6-9 July 2009, Winona Lake, p. 664-682.

_ à paraître : «L'aménagement de l'espace sacré en Mésopotamie du Nord au Bronze moyen, une question de tradition », dans L. Battini (éd.), Quoi de neuf ? Les aménagements au Proche-Orient ancien (IVe-Ir millénaires av. J.-C.), Oxford.

Oren E. D., 1971 : « A Middle Bronze Age I Warrior Tomb at Beth-Shan », ZDPV 87/2, p. 109-139.

1997 : "The "Kingdom of Sharuhen" and the Hyksos Kingdom ", dans

E. D. Oren (éd.), The Hyksos: New Historical and Archaeological Perspectives, Philadelphie, p. 253-283.

Orthmann W., 1981 : Halawa 1977 bis 1979: Vorläufiger Bericht über die 1. bis 3. Grabungskampagne, Saarbrücker Beiträge zur Altertumskunde 31, Bonn.

Philip G., 1995 : «Warrior Burials in the Ancient Near-Eastern Bronze Age: the Evidence from Mesopotamia, Western Iran and Syria-Palestine », dans S. Campbell \& A. Green (éd.), The Archaeology of Death in the Ancient Near East, Oxbow Monograph 51, Exeter, p. 140-154.

2006 : Tell el-Dab'a XV: Metalwork and Metalworking Evidence of the Late Middle Kingdom and the Second Intermediate Period, Vienne.

Piatkowska-Malecka J. \& Z. Wygnańska, 2012 : « Animals Remains from Middle Bronze Age Graves at Tell Arbid (Syria)», Światowit. Annual of the Institute of Archaeology of the University of Warsaw IX, p. 67-80.

Piatkowska-Malecka J. \& A. Smogorzewska, 2010 : « Animal Economy at Tell Arbid, north-east Syria, in the third millennium BC », Bioarchaeology ot the Near East 4, p. 25-43.

Piesl-Trenkwalder H., 1981-1982 : « Tell Ababra 1978-1979 », AfO 28, p. 249252.

Porter A., 2002 : «The Dynamics of Death : Ancestors, Pastoralism, and the Origins of a Third-Millennium City in Syria », BASOR 325, p. 1-36.

Postgate J. N. \& P. J. Watson, 1979 : « Excavations in Iraq, 1977-78 », Iraq 41/2, p. $142-181$.

Rehm E., 2003 : Waffengräber im Alten Orient. Zum Problem der Wertung von Waffen in Gräbern des 3. und frühen 2. Jahrtausends v.Chr. in Mesopotamien und Syrien, BAR Int. Ser. 1191, Oxford.

Roaf M. D. \& J. N. Postgate, 1981 : « Excavations in Iraq, 1979-1987 », Iraq 43, p. 167-198.

Schwartz G., H. Curvers, S. Dunham, B. Stuart \& J. Weber, 2006 : « A ThirdMillennium B.C. Elite Mortuary Complex at Umm el-Marra, Syria: 2002 and 2004 Excavations », AJA 110/4, p. 603-641.

Scurlock J., 2002 : " Animal Sacrifice in Ancient Mesopotamian Religion », dans B. J. Collins (éd.), A History of the animal world in the ancient Near East, Leiden, p. 389-404.

Silver M., 2014 : «Equid Burials in Archaeological Contexts in the Amorite, Hurrian and Hyksos Cultural Intercourse », Aram 26/1\&2, p. 335-355.

Testart A., 2004 : La Servitude volontaire 1 : Les morts d'accompagnement, Paris. 
Tufnell O., 1958 : Lachish IV (tell ed-Duweir). The Bronze Age. WellcomeMarston Archaeological Research Expedition to the Near East I, Oxford.

Tunca Ö., 2012 : « Essai de typologie des tombes ordinaires de l'âge du Bronze ancien et moyen dans la Jezireh orientale en Syrie: le cas de Chagar Bazar et ses implications », dans P. Quenet \& M. Al-Maqdissi (éd.), "L'heure immobile" entre ciel et terre. Mélanges en l'honneur d'Antoine Souleiman, Subartu XXXI, Turnhout, p. 137-155.

Vila E., 2006 : « Data on equids from late fourth and third millennium sites in northern Syria », dans M. Mashkour (éd.), Equids in Time and Space. Papers in Honour of Véra Eisenmann, Oxford, p. 101-123.

Wapnish P., 1997 : " Middle Bronze Age Equid Burials at Tell Jemmeh and Reexamination of a Purportedly "Hyksos" Practice », dans E. D. Oren (éd.), The Hyksos: New Historical and Archaeological Perspectives, Philadelphie, p. 335-367.

Way K. C., 2010 : «Assessing sacred asses: Bronze Age donkey burials in the Near East », Levant 42/2, p. 210-225.

Weber J., 2008 : «Elite equids: redefining equid burials of the mid- to late 3rd millennium BC from Umm el-Marra, Syria », dans E. Vila, L. Gourichon, A.M. Choyoke \& H. Buitenhuis (éd.), Archaeozology of the Near East VIII: Proceedings of the Eight International Symposium on the Archaeozoology of Southeastern Asia and Adjacent Areas, T. II. Actes du 8 colloque international de l'A.S.W.A. tenu à Lyon du 28 juin au $1^{\text {er }}$ juillet 2006, Travaux de la Maison de l'Orient 49, Lyon, p. 499-459.

Wygnańska Z., 2012 : " Mortuary Practices of the End of the Third and of the Early Second Millennium BC on Tell Arbid (Northeastern Syria): Continuity and Changes », in N. Laneri, P. Pfälzner \& S. Valentini (éd.), Looking North. The Socioeconomic Dynamics of Northern Mesopotamian and Anatolian Regions during the Late Third and Early Second Millenium BC, Studien zur Urbanisierung Nordmesopotamiens Serie D, Band I, Wiesbaden, p. 229-236.

Wygnanska Z., 2017 : «Equid and Dog burials in the ritual landscape of Bronze Age Syria and Mesopotamia », Aram 29/1-2, p. 141-160.

Zarins J., 1986 : «Equids Associated with Human Burials in Third Millennium B.C. Mesopotamia: Two Complementary Facets », dans R. Meadow \& H.-P. Uerpmann (éd.), Equids in the Ancient World, volume 1. Beihefte zum Tübinger Atlas des Vorderens Orients Bd. 19/1, Wiesbaden, p. 164-193. 